RESEARCHARTICLE

\title{
The Two-Year Outcomes and Cost Effectiveness of a Commercial Weight Loss Program for the Prevention of Type 2 Diabetes among People with Prediabetes
}

\author{
Kelly N.B. Palmer ${ }^{1 *}$,Chandan Saha ${ }^{2}$, Erin O' Kelly Phillips ${ }^{3}$, Anirudh Krishnan, Gary D. \\ Foster $^{5,6,7}$, Eric Finkelstein ${ }^{4}$, Alexis C. Wojtanowski ${ }^{5}$ and David G. Marrero ${ }^{1}$ \\ 'University of Arizona Health Sciences, USA \\ ${ }^{2}$ Department of Biostatistics, Indiana University School of Medicine, USA \\ ${ }^{3}$ Regenstrief Institute, Inc., USA \\ ${ }^{4}$ Health Services and Systems Research Program, Duke-NUS Medical School, Singapore \\ ${ }^{5}$ Weight Watchers International, Inc., USA \\ ${ }^{6}$ Center for Obesity Research and Education, Temple University, Philadelphia, USA \\ ${ }^{7}$ Center for Weight and Eating Disorders, University of Pennsylvania, Philadelphia, USA
}

\section{๑}

\section{G}

Open Access

Citation: Palmer KNB, Saha C, Phillips EO, Krishnan A, Foster GD, Finkelstein E, Wojtanowski AC, Marrero DG.The Two-Year Outcomes and Cost Effectiveness of a Commercial Weight Loss Program for the Prevention of Type 2 Diabetes among People with Prediabetes. Endocrinology Diabetes and Obesity. 2018; 1(1):5.

Received: February 12, 2018

Accepted: June 18, 2018

Published: June 27, 2018

Copyright: $\odot 2018$ Palmer KNB. This is an open access article distributed under the terms of the Creative Commons Attribution License, which permits unrestricted use, distribution, and reproduction in any medium, provided the original author and source are credited.

Corresponding author:

Kelly N.B. Palmer, University of Arizona Health Sciences, 1295 N Martin Ave, Tucson, AZ 85721, USA

Email: kpalmer1@email.arizona.edu

\section{Abstract}

Objectives: There is solid evidence that risk for developing type 2 diabetes can be prevented by lifestyle interventions. However, there are few diabetes prevention programs available. Given the increasing prevalence of prediabetes, we sought to investigate if a commercial weight loss program with significant capacity could address this need. This study, conducted in Indianapolis, Indiana in 2014-16, investigated if (Weight WatchersWW) could cost effectively achieve and sustain sufficient weight loss in persons with prediabetes to reduce diabetes risk for 24 months.

Methods: A previous, randomized controlled trial evaluated the effectiveness of the WW program in 225 persons with prediabetes as determined by an $\mathrm{HbA} 1 \mathrm{c}$ value $\geq 5.7 \%$ and $\leq 6.4 \%$ or a self-reported history of gestational diabetes with an $\mathrm{HbA} 1 \mathrm{c}<6.5 \%$ and/or casual capillary blood glucose $(C C B G)<199 \mathrm{mg} / \mathrm{dL}$ on weight and metabolic regulation compared with a self-initiated program developed by the National Diabetes Education Program over a 12-month study period. This continuation study assessed outcomes at 18 and 24 months and also evaluated cost effectiveness at 12 and 24 months from a third-party payer perspective. Since this study used a cross over design in which control subjects were provided access to the WW program from 12-24 months, they were no longer randomized.

Results: Intervention participants lost significantly more weight than the controls both at $18(-5.1 \%$ vs $-1.8 \%, p \leq .008)$ - and 24 -months $(-4.5 \%$ vs $-1.8 \%, p \leq .032)$. Although both groups showed some improvement in CVD risk factors, the only significant difference between groups was that WW participants had greater reductions than controls in $\mathrm{HbA} 1 \mathrm{C}$ at both $18(-0.27$ vs- $0.17 ; p=.03)$ and 24 months $(-0.3$ vs $-0.2 ; p=.04)$. Converting the weight loss into quality adjusted life years saved (QALYs) yielded an incremental cost effectiveness ratio (ICER) of $\$ 19,034$ per QALY gained for the intervention. Sensitivity analyses showed the ICER was well below commonly accepted thresholds for cost effectiveness.

Conclusion: These data suggest that evidence-based, widely available weight management programs have the potential to cost effectively improve health outcomes for patients with prediabetes. Given their affordability and scalability, increasing access could result in a significant public health impact. 


\section{Keywords}

Type 2 diabetes, prediabetes, weight loss

\section{Introduction}

Over 84 million adults in the United States have prediabetes. ${ }^{1}$ Without effective intervention strategies, $5-10 \%$ of these individuals will go on to develop diabetes each year. ${ }^{2}$ This $^{2}$ has stimulated the development of community-based diabetes prevention programs most of which are implemented by the Young Men's Christian Association (YMCA) or programs that have been trained by the National Diabetes Prevention Program (NDPP). ${ }^{3,4}$ This is an important advance, however, there remain far too few facilities to meet the growing demand. As a result, there is a significant need for increased access to low cost, scalable, evidencebased diabetes prevention programs.

In 2016, we reported the results of a randomized control trial demonstrating that a nationally available weight loss program, Weight Watchers $₫(W W)$, achieved sustained weight loss over a 12-month period among a sample of those with prediabetes, consistent with the weight losses in the Diabetes Prevention Program (DPP) study. ${ }^{5,6}$ Here we report weight and glycemic outcomes and cost effectiveness over an 18 and 24 months period to further illuminate the potential for commercial weight loss programs to better meet the need for diabetes prevention programs. We sought to determine if WW could produce sustained weight loss in a cost-effective manner.

\section{Methods}

\section{Design}

Initially, we conducted a randomized controlled trial comparing the evidence-based WW meeting program with a self-initiated program developed by the National Diabetes Education Program (NDEP) on weight and glucose control as measured by hemoglobin A1C (HbA1c). ${ }^{7,8}$ Participants were recruited via flyers mailed to homes proximal to WW facilities, company list serves, health fairs, community sites such as churches, and by participant referrals. After the 12-month study period, participants originally assigned to the WW program were allowed to continue participation in WW for an additional 12 months for free, for a total of 24 months. Those in the self-guided group, the wait list control group, were also provided free access to WW for the next 12 months. Because our primary outcome measure from the initial trial was percent weight change from baseline to 6 and 12 months, for the second year of the study we wanted to focus on percent weight change from baseline to 18 and 24 months (continued or sustained weight loss for those in the intervention). We were also interested in what effect, if any, delaying access to the intervention, but providing guidance for self-initiated weight loss would have on weight loss for those in the wait list control group. Research assistants were trained by the Principal Investigator to deliver instruction on the self-initiated program to study participants.

The WW intervention was delivered by appropriately trained group leaders, all of whom were successful on the WW program themselves. The WW curriculum is delivered in a group environment using a "loop" delivery model (repeating themes) that addresses the same behavioral topics used in the DPP: 1) self-monitoring of weight, intake and activity; 2) dietary modification; 3) physical activity; 4) stimulus control; and 5) relapse prevention. Before beginning the 12-month lifestyle intervention program, participants attended a 45-minute "activation" session at one of 5 WW facilities in the Indianapolis area. This was done within one week of the baseline assessment. This session was conducted by existing WW leaders who were trained for this study-specific session. It focused on educating participants about the meaning of prediabetes, how the condition increases risk for developing type 2 diabetes, and the role of lifestyle modification to reduce their risk. A weight loss goal of $7 \%$ was also assigned. Following this activation session, participants were enrolled into the already existing WW program in the community. Each participant was free to choose a WW group session time and location that was convenient for them. Participants attended already existing meetings that were not study-specific and included those without prediabetes. They were encouraged to attend the weekly meetings and told they were free to switch both time and location as best fit their schedules. They were also given access to the WW e-tools, which 
includes digital tools to track weight, intake and activity as well as tips to facilitate adherence.

Participants assigned to the control condition were provided a review of how they could initiate a weight loss and activity program using Your Game Plan to Prevent Type 2 Diabetes educational materials developed by the National Diabetes Education Program. ${ }^{7,8}$ These materials review the meaning and implications of prediabetes, the results of the DPP study, an overview of how to initiate a risk reducing lifestyle program, a reproducible tracker to help monitor their food intake, and a booklet with fat gram and calorie content for several common foods. Emphasis was placed on strategies for tracking food intake and calculating fat grams using the food tracker and calorie fat gram guide provided in the materials. This individual counseling, which took approximately 15 minutes, was provided by trained research staff and conducted during the screening event after randomization. During the follow up data collection visit at 12 months, wait list control participants were offered the WW intervention. Research staff activated WW memberships for those opting to enroll in the intervention. Control participants were not offered the "activation session" the original intervention participants attended, but rather folded directly into the existing WW program (meetings and e Tools). The "activation session" was deemed not necessary for the control participants because they were exposed to the self-guided program using the NDEP materials for a year and were aware of their risk for developing diabetes.

Indiana University Institutional Review Board approval was obtained for the study protocol. The Clinical Trials Registration number is NCT02000024. All participants provided written informed consent and were provided copies of the consent forms stating study objectives.

\section{Participants}

The eligibility and ineligibility criteria for participation have been described previously. ${ }^{5}$ In summary, a person had to be age 18 or greater, have a body mass index $(\mathrm{BMI}) \geq 24 \mathrm{~kg} / \mathrm{m}^{2}$ (persons of Asian Descent BMI $>23 \mathrm{~kg} / \mathrm{m}^{2}$ ), and score $\geq 5$ on the 7-item American Diabetes Association (ADA) Diabetes Risk Assessment. ${ }^{9}$ In addition, they had to have prediabetes as determined by an $\mathrm{HbA} 1 \mathrm{c}$ value $\geq 5.7 \%$ and $\leq 6.4 \% .10$ Women with a self-reported history of gestational diabetes with an $\mathrm{HbA} 1 \mathrm{c}<6.5 \%$ and/or casual capillary blood glucose $(\mathrm{CCBG})<199 \mathrm{mg} / \mathrm{dL}$ were also included. ${ }^{10,11}$

To determine prediabetes status, screening sessions were held at community sites to collect $\mathrm{HbA} 1 \mathrm{c}, \mathrm{CCBG}$, and the ADA risk assessment following the equipment and protocol described in the first paper. ${ }^{5}$

\section{Measures}

Measures were collected by an Indiana University School of Medicine research team member at community sites after 18 and 24 months of study enrollment following the protocol described in our first outcomes paper. ${ }^{5}$ The primary outcome was percent change in body weight at 18 and 24 months. Secondary outcomes included changes in blood pressure, $\mathrm{HbA1c}$, total cholesterol, and high-density lipoprotein-cholesterol (HDL-c).

\section{Randomization}

At the beginning of this study we recruited and screened 475 persons. 225 eligible and consented participants were randomly assigned either to the intervention or the control group in $1: 1$ ratio using a computer-generated randomization list with a block size of 4 . All participants received personalized advice about their risk for developing diabetes and were advised that modest weight loss $(5 \%-10 \%)$ via caloric restriction and the adoption of moderate physical activity were generally safe and effective in preventing or delaying the onset of diabetes. The 112 intervention group participants were randomized to and offered the WW program. $83.9 \%$ of the intervention participants activated and enrolled in the WW intervention in the first year of the study. 113 controls were provided counseling regarding selfinitiated weight loss using materials developed by the National Diabetes Education Program ${ }^{7,8}$ and informed that they would be provided access to the WW intervention after 12 months. Thus, they constituted a "wait list control." $48.9 \%$ of the wait list controls activated and enrolled in the WW intervention in the second year of the study. In this paper we report the long-term data from intervention and control subjects who continued in the study with many of the controls having elected to participate in WW from months 12 to 24.

\section{Data Analyses}

Descriptive statistics were used to summarize all the baseline continuous variables as a 
whole was and presented by treatment group. Frequency counts and percentages were used to summarize all the baseline categorical variables. We conducted an intention to treat analysis even though there was a crossover at 12 months that allowed participants in the control condition to be exposed to the intervention treatment. Tables 1 and 2 in the results section show the number of participants in each group after the 12-month primary analysis in which control participants could utilize the WW program. Baseline clinical and demographic data were compared between the two groups including the participants who had the 24-month visit. Chi-square tests or Fishers exact test were used to examine dichotomous and ordinal variables and two-sample t-test or two sample non-parametric Wilcoxon Rank Sum Test (if normality assumptions were not met) were used to examine continuous variables. The percent weight changes at 18 and 24 months from baseline were defined as the primary outcomes. Repeated measures ANCOVA was used to analyze the longitudinal data on percent weight change at 18- and 24- month from baseline. Secondary outcomes were changes in $\mathrm{HbA} 1 \mathrm{C}$, blood pressure, total cholesterol, and HDL at 18 and 24 months. Again, repeated measures ANCOVA was used to analyze all secondary outcomes.

Table 1. Baseline characteristics of those who completed the 24-month visit

\begin{tabular}{|c|c|c|c|}
\hline Characteristics & $\begin{array}{r}\text { Control } \\
(n=72)\end{array}$ & $\begin{array}{c}\text { Intervention } \\
(n=85)\end{array}$ & P-value \\
\hline \multicolumn{4}{|l|}{ Demographics } \\
\hline Age in years, mean (SD) & $52.2(11.0)$ & $52.5(11.0)$ & .88 \\
\hline Gender, \% female & 86.1 & 83.5 & .82 \\
\hline Ethnicity, \% non-Hispanic & 92.9 & 92.9 & .73 \\
\hline $\begin{array}{l}\text { Race, } \% \\
\text { Caucasian } \\
\text { African American } \\
\text { Asian/Pacific Islander } \\
\text { Multi-racial } \\
\text { Other }\end{array}$ & $\begin{array}{r}63.9 \\
20.8 \\
9.7 \\
1.4 \\
4.2\end{array}$ & $\begin{array}{r}60.0 \\
32.9 \\
4.7 \\
1.2 \\
1.2\end{array}$ & .26 \\
\hline $\begin{array}{l}\text { Education, \% } \\
\text { Some high school } \\
\text { High school graduate or GED } \\
\text { Some college or } 2 \text {-yr college } \\
\text { degree } \\
\text { 4-yr college graduate } \\
\text { More than 4-yr college degree }\end{array}$ & $\begin{array}{c}2.9 \\
5.8 \\
30.4 \\
23.2 \\
37.7\end{array}$ & $\begin{array}{c}2.3 \\
7.1 \\
34.1 \\
18.8 \\
37.6\end{array}$ & .96 \\
\hline $\begin{array}{l}\text { Marital status, \% } \\
\text { Married } \\
\text { Divorced } \\
\text { Never married } \\
\text { Widowed } \\
\text { Separated } \\
\text { Living with partner }\end{array}$ & $\begin{array}{r}57.1 \\
25.7 \\
10.0 \\
2.9 \\
1.4 \\
2.9\end{array}$ & $\begin{array}{r}60.0 \\
20.0 \\
14.1 \\
4.7 \\
0.0 \\
1.2\end{array}$ & .71 \\
\hline $\begin{array}{l}\text { Income, \% } \\
<\$ 10 \mathrm{~K} \\
\$ 10 \mathrm{~K}-\$ 15 \mathrm{~K} \\
\$ 15 \mathrm{~K}-\$ 25 \mathrm{~K} \\
\$ 25 \mathrm{~K}-\$ 35 \mathrm{~K} \\
\$ 35 \mathrm{~K}-\$ 50 \mathrm{~K} \\
\$ 50 \mathrm{~K}-\$ 75 \mathrm{~K} \\
>\$ 75 \mathrm{~K} \\
\text { Didn't know } \\
\quad \text { Refused } \\
\end{array}$ & $\begin{array}{r}0.0 \\
2.9 \\
0.0 \\
10.0 \\
18.6 \\
24.3 \\
34.3 \\
2.9 \\
7.1 \\
\end{array}$ & $\begin{array}{r}2.3 \\
2.3 \\
5.9 \\
10.6 \\
17.6 \\
17.6 \\
35.3 \\
3.5 \\
4.7 \\
\end{array}$ & .56 \\
\hline Clinical Variables & Mean (SD) & Mean (SD) & \\
\hline Body mass index, $\mathrm{kg} / \mathrm{m}^{2}$ & $35.9(6.7)$ & $36.8(7.1)$ & .41 \\
\hline A1c, \% & $5.8(0.4)$ & $5.9(0.3)$ & .052 \\
\hline Glucose, mg/dL & $109.9(20.2)$ & $106.9(22.1)$ & .38 \\
\hline Total cholesterol, mg/dL & $197.9(41.3)$ & $187.0(31.9)$ & .06 \\
\hline $\mathrm{HDL}, \mathrm{mg} / \mathrm{dL}$ & $49.4(14.2)$ & $48.8(15.0)$ & .78 \\
\hline Diastolic blood pressure, $\mathrm{mmHg}$ & $80.1(8.2)$ & $82.6(8.7)$ & .07 \\
\hline Systolic blood pressure, $\mathrm{mmHg}$ & $130.0(14.7)$ & $130.3(12.3)$ & .88 \\
\hline Weight, $\mathrm{kg}$ & $97.5(19.1)$ & $100.0(20.4)$ & .41 \\
\hline
\end{tabular}


Table 2. Changes in body weight and clinical outcomes at 18 and 24 months after adjusted for baseline values of outcomes

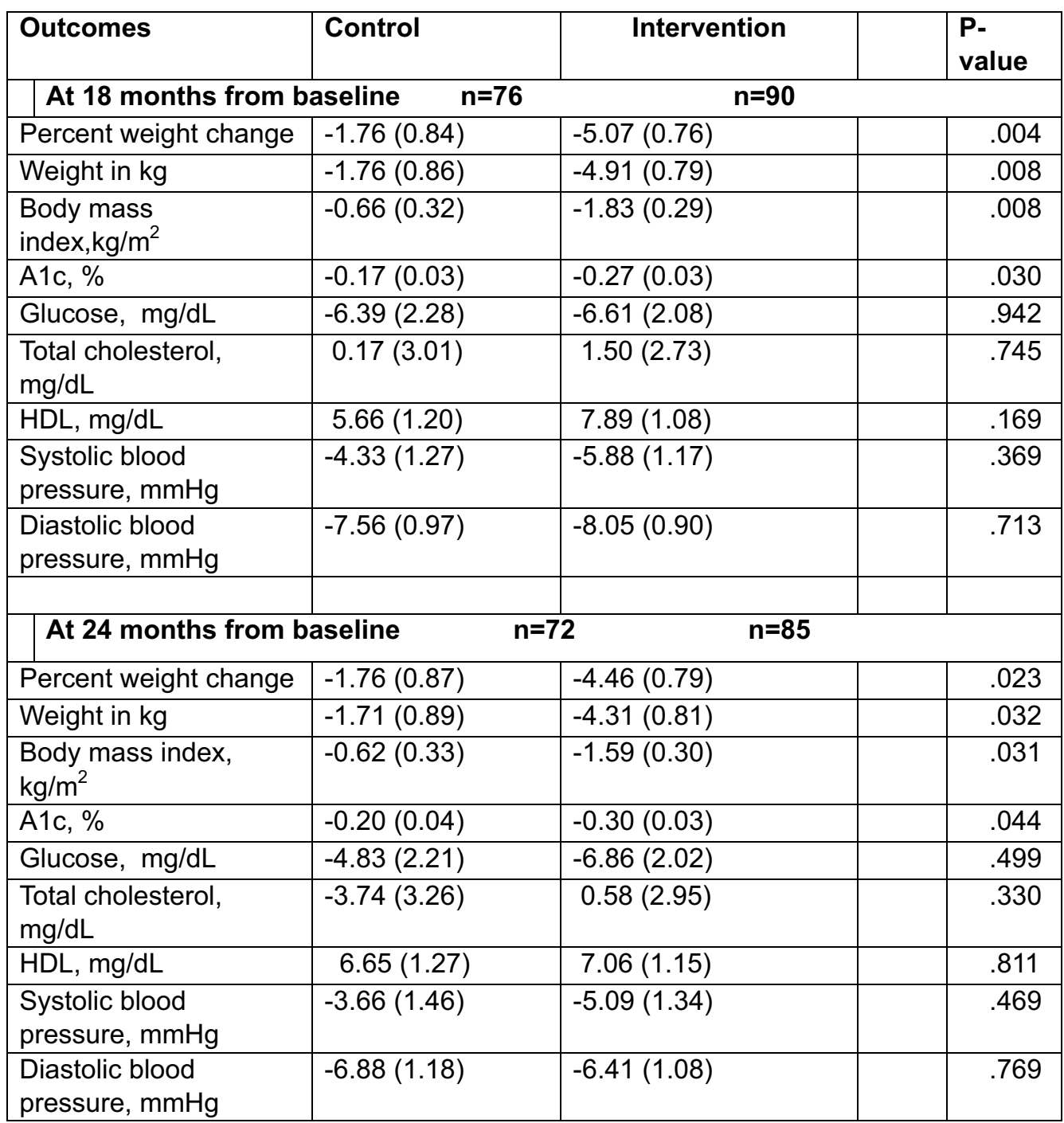

Values are means (SE) adjusted for baseline value of the outcomes.

In addition, we analyzed the proportion of participants with at least $5 \%$ weight loss as well as the proportion of participants with at least $7 \%$ weight loss at 18 - and 24 -month. We used logistic regression model with generalized estimating equations (GEE). All models included baseline value of the outcome variable. An unstructured covariance matrix was used in all models. We performed sensitivity analysis to account for missing weight data (33\% at 18 months and $36 \%$ at 24 months in control group; $20 \%$ at 18 months and $24 \%$ at 24 months in the intervention group). Sensitivity analyses included multiple imputation method with the assumption that missing data at follow-up was a function of treatment group and baseline weight. The only difference among those who had or did not have weight data at 24 months was that those who had missing weight data at 24 months had a significantly higher baseline weight than those with non-missing weight data both in the control and intervention groups. There was, however, no significant difference between the intervention and control groups in baseline weight.

\section{Cost Effectiveness Analysis}

We conducted a cost effectiveness analysis from the societal perspective. It included market prices for participants to access the program but did not include any potential cost offsets that may come from improved health. The market price was a reduced rate offered to employers who offered the WW program as a benefit to their employees, representing a reduction of $15 \%$ from the individual subscription rate. All participants randomized to the WW arm were assigned this cost in year one. In year two, a second year of costs was assigned to those WW participants who accessed the program at least once. Participants in the control 
arm were assigned zero cost for year one. For the second year, we applied the annual cost of the WW program to control participants who accessed the WW program at least once, as the design allowed for control participants to cross over into the intervention arm in year two should they wish. All year two costs were discounted to baseline at a rate of $3.5 \%$ per annum.

The primary measure of effectiveness was an imputed quality of life (QoL) change score using the data and methods described in Finkelstein, Kruger and Karnawat and Finkelstein and Kruger ${ }^{12,13}$ but with the sample restricted to participants whose characteristics are most similar to the current study population. The restricted sample included participants with BMI $>=24 \mathrm{~kg} / \mathrm{m}^{2}, \mathrm{~A} 1 \mathrm{c}$ levels between $5.7 \%$ and $6.5 \%$ (inclusive), systolic blood pressure $<=180$ $\mathrm{mmHg}$ and diastolic blood pressure $<=105 \mathrm{mmHg}$. Using this sample and regression strategy we estimated the association between QoL change and weight change as follows: QoL change $=-0.002 \times$ weight change $($ in $\mathrm{kg})+0.01$. Using this equation, we estimated QoL changes for each individual at each assessment point with respect to baseline.

The incremental cost effectiveness ratio was calculated by dividing the average incremental cost of the intervention by the average incremental effectiveness. The numerator is the mean incremental discounted cost of the WW intervention with respect to the control arm. The denominator is the mean discounted Quality-Adjusted Life Years (QALYs) gained in the treatment arm less discounted QALYs gained by the control arm, where QALYs are equal to the QoL weight times the duration (years) that the weight loss benefits are expected to accrue. All out-year cost and QALY estimates were discounted at $3.5 \%$ per annum.

Two separate cases were evaluated. The base case analyzed cost and effectiveness for the entire 2-year intervention period. However, as $76.4 \%$ (55) of the 72 participants in the control group who remained in the study at 24-month, received the WW program in year two, this influenced the cost and QALYs of the 'controls', which is likely to have biased the incremental effectiveness of WW towards the null. Therefore, we also conducted a second analysis based solely on the data at the end of year one. We estimated the ICER for each case making the following assumptions: (1) QoL benefits from weight loss accrued at 50\% during the first year of the intervention (to account for lags in weight loss and the corresponding improvement in QoL); (2) QoL benefits accrued at 100\% in the second year of the intervention; and (3) benefits linearly decay to 0 by the end of the second year, post cessation of the intervention in both base cases.

Sensitivity Analyses. To test for the sensitivity of our ICER estimates to changes in key inputs, for both the one and two-year analyses we conducted one-way sensitivity analyses by (1) doubling or halving the incremental cost of the intervention with respect to control; (2) doubling or halving the incremental QALYs with respect to control; and (3)varying the duration of benefit decay post program cessation from 2 years in both base cases to 0,1 , and 3 years.

\section{Results}

Table 1 shows comparisons of baseline characteristics between the intervention and control groups including the participants who had the 24-month visit. Overall, the two groups were similar in baseline demographic and clinical variables. Table 2 depicts comparisons of changes in primary and secondary outcomes at 18- and 24-month from baseline (month 0 ). The WW intervention group lost significantly more weight than the control group both at 18 and 24 months. The mean percent weight loss was more than two and a half times higher in the intervention group compared with the control group. As previously reported, the mean percent weight loss in the intervention group was 5.5\% at 6 - and 12 -month, compared to less than $1 \%$ in the control group. ${ }^{5}$ The maintained weight loss at 18 - and 24 -months was $92 \%(5.07 \%$ weight loss) and $81 \%(4.46 \%)$ of the 6 - and 12-month weight losses, respectively. ${ }^{5}$ The control group showed several folds greater weight loss at 18- and 24-month compared to the negligible weight loss at 6 - and 12-months likely because almost half (48.9\%) of the control subjects participated in the WW intervention after 12-months. The sensitivity analyses for treatment difference on percent weight change showed almost the same results at 18-months and 24-months. Specifically, the mean treatment differences (SE) without simulation were $3.3 \%(1.1 \%)$ at 18 -months and $2.7 \%(1.2 \%) 24$-months versus simulation values $3.6 \%(1.2 \%)$ at 18 -months and $2.9 \%(1.3 \%) 24$ months.

A greater proportion of those in the intervention group (41\%) lost $>7 \%$ of initial body weight compared to the control group(28\%)at both 18-and 24-months, though the difference did not reach statistical significance (18-month: $p=.055$, 24-month: $p=.112)$. Similarly, the 
intervention group had 1.8 times higher odds of having at least $7 \%$ weight loss at 18 -months and 1.6 times higher odds at 24-months compared to the control group, though again the difference did not reach statistical significance (18-month: $p=.071,24-$ month: $p=.201)$.

Although both groups showed improvement in cardiovascular disease (CVD) risk factors, the only significant difference between groups was that Intervention participants had greater reductions than controls in $\mathrm{HbA} 1 \mathrm{c}$ at $18(-0.27$ vs. $-0.17 ; p=.03)$ and 24 months $(-0.3$ vs -0.2 ; $p=.04)$.

\section{Cost Effectiveness Analysis}

In year one, $83.9 \%$ (94) of the 112 participants randomized into the WW intervention accessed the program. In year two, $60.0 \%$ (51 of 85) of the WW participants who did not drop out of the intervention accessed the program and $76.4 \%$ (55 of 72 ) of the remaining control group participants also crossed over and accessed the WW intervention.

The annual cost of the intervention was $\$ 438$ per participant in the first year, and either $\$ 438$ or $\$ 459$ per participant in the second year, depending on date of activation. Mean incremental cost was $\$ 439$ in the one-year base case and $\$ 369$ in the two-year case, where the lower cost reflected the reality that $40 \%$ (34 of 85 ) participants remaining in the intervention had zero costs in year two because they did not access the program. The greater weight loss in the WW arm resulted in larger improvements in imputed QoL scores for intervention participants compared to the control group at 12 months $(+0.021$ versus +0.011$), 18$ months $(+0.020$ versus $+0.014[p=0.008])$, and at 24 months $(+0.019$ versus $+0.014[p=0.032])$.

Using the full 2-year intervention period with linear decay of post-intervention benefits within 2 years, we estimated an ICER of $\$ 19,034.05$ per QALY gained. The one-year base-case assumption yielded an ICER of $\$ 24,739$ per QALY gained. Figure 1 presents the results of one-way sensitivity analyses around the 2-year analysis. Doubling incremental costs or halving incremental QALYs resulted in an ICER of $\$ 38,068$ per QALY; and halving incremental costs or doubling incremental QALYs decreased the ICER to $\$ 9,517$ per QALY. With no posttrial benefits, the ICER rose to $\$ 25,513$; and with a $3-y e a r$ linear decay of post-trial benefits, the ICER fell to $\$ 17,640$.

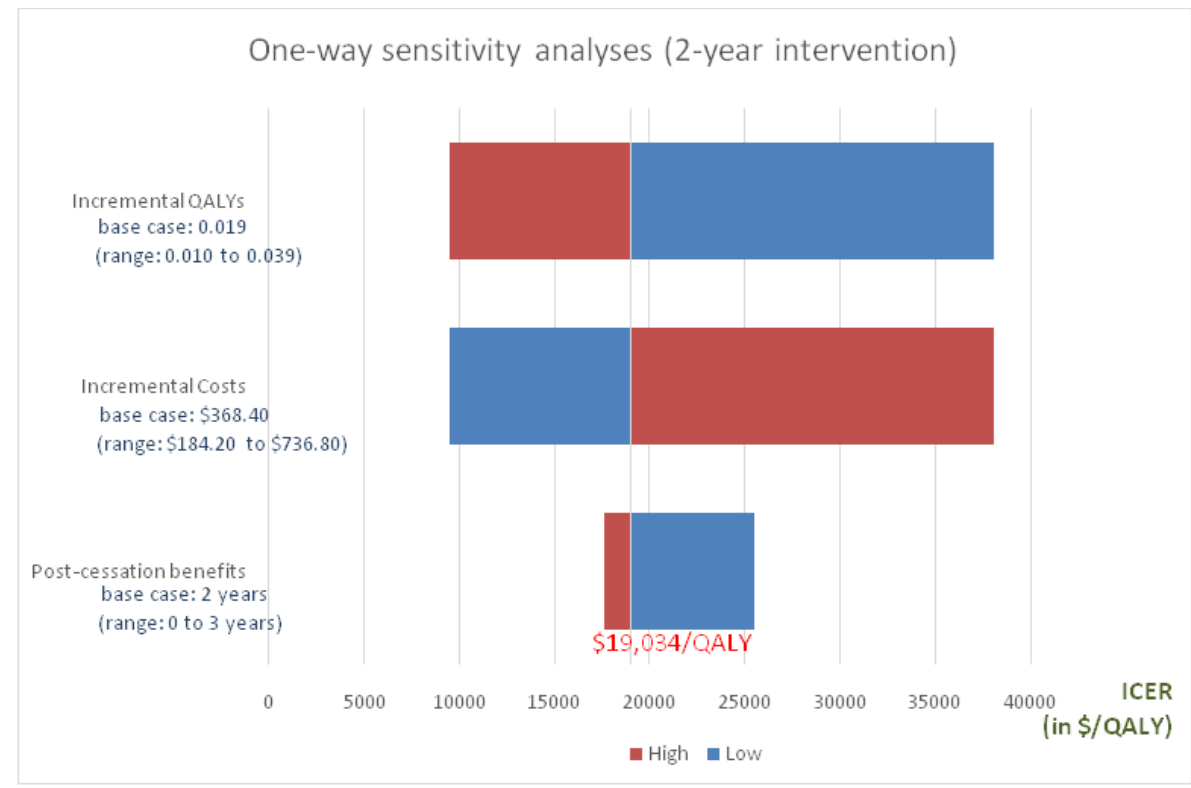

Figure 1. Tornado diagram presenting results of one-way sensitivity analyses in the 2-year case: Each horizontal bar represents sensitivity of the ICER to changes in the corresponding variable presented to its left. High and low values represent double and half the base case value of each incremental QALYs and incremental costs; and 3 and 0 years respectively for post-cessation benefits

In the one-year base case analysis, doubling incremental costs or halving incremental QALYs resulted in an ICER of $\$ 49,479$ per QALY. Figure 2 presents results of sensitivity analyses around the 1-year base case. Halving the incremental costs or doubling the incremental QALYs decreased the ICER to $\$ 12,370$ per QALY. Decreasing post-trial benefits to 0 years produced an ICER of $\$ 59,426$. With three years of benefits post-cessation of the intervention, the ICER fell to $\$ 19,285$. 


\section{One-way sensitivity analyses (1-year intervention)}

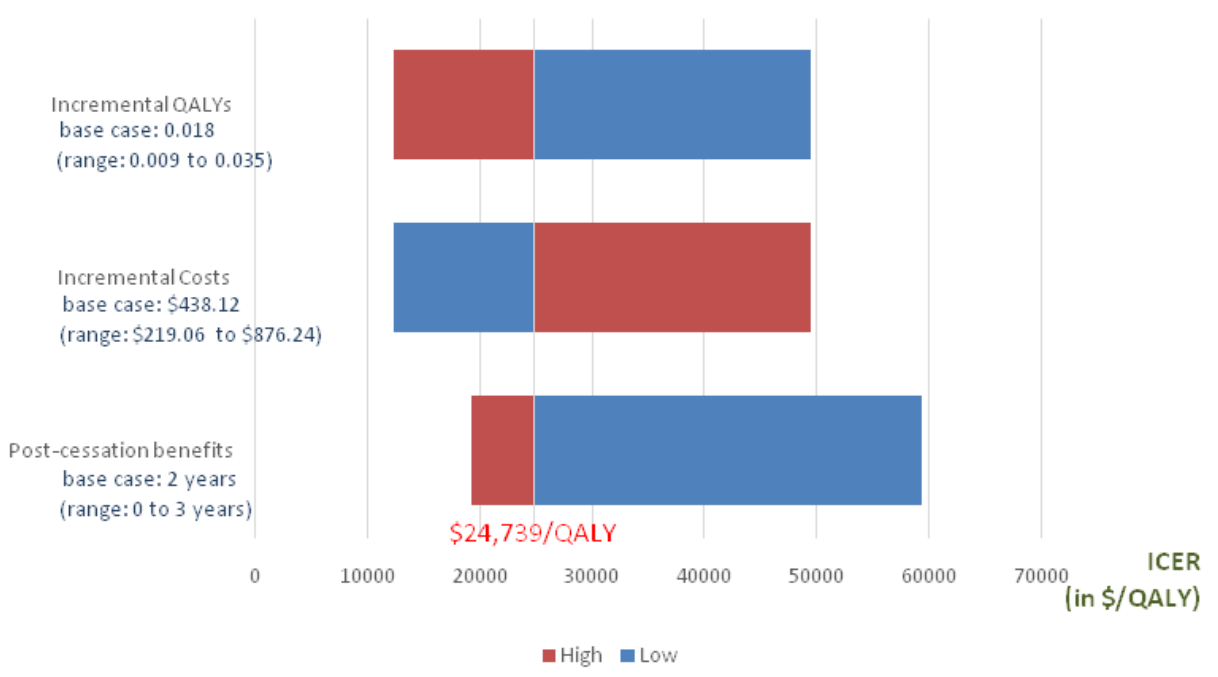

Figure 2. Tornado diagram presenting results of one-way sensitivity analyses in the 1-year case: Each horizontal bar represents sensitivity of the ICER to changes in the corresponding variable presented to its left. High and low values represent double and half the base case value of each incremental QALYs and incremental costs; and 3 and 0 years respectively for post-cessation benefits

\section{Discussion}

Diabetes is rapidly becoming a major and growing public health crisis worldwide, with increasing medical, social, and fiscal impact. It is ironic that in spite of substantial evidence that lifestyle interventions for weight loss can significantly reduce type 2 diabetes risk in persons with prediabetes, there are relatively few evidence-based programs available at scale. While diabetes prevention programs are on the increase, there are still far too few to meet the current and expanding public health need. The data from this study show that a widely available weight management program results in changes in body weight after 18 and 24 months that are comparable to those observed in the DPP study and community adaptations $^{15-19}$. The program studied here, Weight Watchers, is an evidence-based lifestyle intervention that combines face to face and digital offerings. Importantly, it is widely available with more than 32,000 WW meetings held each week in the U.S., led by trained facilitators in a variety of times and locations, including over 5000 in workplace settings. Thus, WW has the potential for rapidly expanding access to a lifestyle intervention for those with prediabetes that significantly exceeds the reach of current efforts.

Cost is an important consideration for scaling up diabetes prevention programs. To explore this, we conducted a cost effectiveness analysis from the perspective of a third-party payer. We chose this view in light of the decision by Centers for Medicare and Medicaid Services (CMS) in 2017 to provide coverage for participation in diabetes prevention programs as a benefit. Our results showed the program is relatively low cost, with annual costs less than $\$ 500$ per year, and highly cost effective based on the commonly cited threshold of $\$ 50,000$ per QALY gained as a measure of what constitutes a cost-effective intervention. ${ }^{20}$

The landscape for providing third party coverage is in transition, with greater likelihood that other payers will follow CMS's example and start providing benefit coverage. Indeed, there are already insurance companies that offer diabetes prevention as a covered benefit. To date, however, all such companies, including CMS, focus upon the 16-session curriculum originated for the Diabetes Prevention Program (DPP) and adapted for use by both the YMCA and the NDPP. This evidenced-based approach, while effective in reducing risk status, has limitations that can impact participation. ${ }^{6,18}$ One important one is the way that program delivery is organized. The standard DPP approach uses sequential session curricula in which each session is only offered once. If you miss a session, it is difficult to make it up. The WW approach uses a "loop" model in which sessions are repeated frequently and at a wide range of times and locations within the same week. In this regard, the program is easy to restart when needed if life events interrupt program attendance. This feature may offer important 
alternatives to consumers in comparison to the traditional DPP format. The WW approach also allows interested parties to join at any time rather than a cohort model which requires a threshold number of people with prediabetes to enroll in a group at a specific day and time. Moreover, WW participants can also access eTools and a mobile app to reinforce session lessons and/or serve as an alternative to in-person meetings. Which further increases access.

The lack of differences between the two groups in lipids and blood pressure is surprising given the well documented effects of weight loss on CVD risk factors, although both groups experienced clinically significant improvements. The lack of group differences may result in part from the large number of control participants who enrolled in the intervention during the second year. There was, however, a significantly greater reduction in $\mathrm{HbA} 1 \mathrm{c}$ among intervention versus controls in spite of randomization being eliminated in year two, though the groups difference was not clinically meaningful. This is consistent with the effects of weight loss on HbA1c among those with prediabetes. It is also possible that the lack of differences observed may result from the reduced emphasis on physical activity by WW in contrast to the DPP approach that sets specific activity goals.

There were several limitations to this study. There was a loss of participants to follow-up at $18(20-33 \%)$ and 24 months (24-36\%). Thus, for the primary weight-loss outcome, the effectiveness of the intervention may have been over-estimated if weight loss was lower in non-completers. There was, however, less attrition in the intervention (20\% at 18 -month, $24 \%$ at 24-month) versus control (33\% at 18-month, 36\% 24-month) groups. Since loss to follow up was greater in the control group, the group differences observed might have been even greater if all participants were represented. Sensitivity analyses on percent weight change showed the very similar results.

Quality of life scores were imputed using data from participants in another study that closely resembled participants in this study. ${ }^{12,13}$ In addition, the majority of the sample were women and had higher socioeconomic status (SES) and education status. Recruitment targeted all residents of the Indianapolis metropolitan area and thus representative of the local demographics (majority Caucasian and female). ${ }^{21}$ Thus, there was no attempt to exclude men or any group on the basis of their SES. This may reflect a tendency of women to gravitate towards weight management programs. ${ }^{22}$ It does caution generalizing the results of this study to low SES populations and men.

These data suggest that Weight Watchers, a nationally available, empirically validated weight management program represents a cost-effective strategy to significantly expand access to diabetes prevention programs in community settings and produce weight-loss levels that translate into considerable reductions in diabetes risk. ${ }^{23}$

\section{References}

1. Centers for Disease Control and Prevention. National diabetes statistics report: estimates of diabetes and its burden in the United States, 2017. Atlanta, GA: US Department of Health and Human Services, Centers for Disease Control and Prevention; 2017.

2. TabákAG, Herder C, Rathmann W, Brunner EJ, Kivimäki M. Prediabetes: a high-risk state for diabetes development. The Lancet. 2012;379:2279-2290.

3. National Diabetes Prevention Program. cdc.gov. https://www.cdc.gov/diabetes/prevention/index.html. Updated January 16, 2018. Accessed May 8, 2018.

4. YMCA's Diabetes Prevention Program. ymca.net. http://www.ymca.net/diabetes-prevention. Accessed May 8, 2018.

5. Marrero DG, Palmer KNB, Phillips EO, Miller-Kovach K, Foster GD, Saha CK. Comparison of commercial and self-initiated weight loss programs in people with prediabetes: a randomized control trial. Am J Public Health. 2016;106(5):949-956.

6. Diabetes Prevention Program Research Group. Reduction in the incidence of type 2 diabetes with lifestyle intervention or metformin. N Engl J Med. 2002;346(6):393-403.

7. National Diabetes Education Program. niddk.nih.gov. https://www.niddk.nih.gov/health-information/ communication-programs/ndep. Accessed May 8, 2018. 
8. Clark CM, Fradkin JE, Hiss RG, Lorenz RA, Vinicor F, Warren-Boulton E. The National Diabetes Education Program, Changing the Way Diabetes Is Treated Comprehensive diabetes care. Diabetes Care. 2001;24:617-618.

9. Herman WH, Smith PJ, Thompson TJ, Engelgau MM, Aubert RE. A new and simple questionnaire to identify people at increased risk for undiagnosed diabetes. Diabetes Care. 1995;18:382-387.

10. Shemesh T, Rowley KG, Shephard M, Piers LS, O'Dea K. Agreement between laboratory results and on-site pathology testing using Bayer DCA2000+ and Cholestech LDX point-of-care methods in remote Australian Aboriginal communities. ClinChimActa. 2006;367:69-76.

11. Dai K-S, Tai D-Y, Ho P, et al. Accuracy of the Easy Touch blood glucose self-monitoring system: a study of 516 cases. ClinChimActa. 2004;349:135-141.

12. Finkelstein EA, KrugerE, KarnawatS. Cost-effectiveness analysis of Qsymia for weight loss. Pharmacoeconomics. 2015;33(7):699-706.

13. Finkelstein EA, KrugerE. Meta- and cost-effectiveness analysis of commercial weight loss strategies. Obesity. 2014;22:1942-1951.

14. Geiss LS, James C, Gregg EW, Albright A, Williamson DF, Cowie CC. Diabetes risk reduction behaviors among US adults with prediabetes. Am J Prev Med. 2010;38(4):403-409.

15. Johnston CA, Rost S, Miller-Kovach K, Moreno JP, Foreyt JP. A randomized controlled trial of a community-based behavioral counseling program. Am J Med. 2013;126: 1143.e19-1143.e24.

16. Ackermann RT, Marrero DG. Adapting the Diabetes Prevention Program Lifestyle Intervention for Delivery in the Community. Diabetes Educ. 2007;33:69-78.

17. Ali MK, Echouffo-Tcheugui JB, Williamson DF. How effective were lifestyle interventions in real-world settings that were modeled on the Diabetes Prevention Program? Health Aff. 2012;31:67-75.

18. Amundson HA, Butcher MK, Gohdes D, et al. Translating the Diabetes Prevention Program into Practice in the General Community Findings from the Montana Cardiovascular Disease and Diabetes Prevention Program. Diabetes Educ. 2009;35:209-223.

19. Jackson L. Translating the Diabetes Prevention Program into Practice A Review of Community Interventions. Diabetes Educ. 2009;35:309-320.

20. Marsille E, Larson B, Kazi DS, Kahn JG, Rosen S. Thresholds for the cost-effectiveness of interventions: alternative approaches. Bulletin of the World Health Organization; 2015. 93:118-124.

21. Indianapolis city (balance), Indiana. factfinder. census.gov. https://factfinder.census.gov/ faces/nav/jsf/pages/community_facts.xhtml?src=bkmk. Accessed May 8, 2018.

22. Millstein RA, Carlson SA, Fulton JE, et al. Relationships between body size satisfaction and weight control practices among US adults. Medscape J Med. 2008;10(5):119.

23. Wing RR. Treatment options for obesity: do commercial weight loss programs have a role? JAMA. 2010;304:1837-1838. 